Itinéraires Itinéraires

Littérature, textes, cultures

2016-3 | 2017

«Banlieues » : entre imaginaires et expériences

\title{
Traverser les banlieues littéraires : entre sensationnalisme et banalité quotidienne
}

Throughout Literary Banlieues: Between Sensationalism and Banality

Serena Cello

\section{OpenEdition}

Journals

Édition électronique

URL : http://journals.openedition.org/itineraires/3595

DOI : $10.4000 /$ itineraires.3595

ISSN : 2427-920X

Éditeur

Pléiade

Référence électronique

Serena Cello, «Traverser les banlieues littéraires : entre sensationnalisme et banalité quotidienne », Itinéraires [En ligne], 2016-3 | 2017, mis en ligne le 15 juillet 2017, consulté le 19 avril 2019. URL : http:// journals.openedition.org/itineraires/3595; DOI : 10.4000/itineraires.3595

Ce document a été généré automatiquement le 19 avril 2019

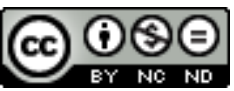

Itinéraires est mis à disposition selon les termes de la licence Creative Commons Attribution - Pas d'Utilisation Commerciale - Pas de Modification 4.0 International. 


\title{
Traverser les banlieues littéraires : entre sensationnalisme et banalité quotidienne
}

\author{
Throughout Literary Banlieues: Between Sensationalism and Banality
}

\author{
Serena Cello
}

1 Les banlieues, entendues comme quartiers populaires sensibles, qui ont été l'objet des débats médiatiques et politiques, notamment depuis les premières émeutes urbaines médiatisées des années 1990 jusqu'aux plus violentes en 2005, semblent rassembler autour d'elles et de leur jeunesse surtout, les connotations les plus disparates et péjoratives : paupérisation socio-économique, ségrégation résidentielle, dégradation du bâtiment, violence et délinquance, malaise, jusqu'à devenir l'« épicentre du problème social contemporain » (Vieillard-Baron 2011).

2 La situation délicate des banlieues lors des révoltes de 2005 aurait été la condition indispensable ayant permis l'essor d'une production romanesque émergente l'année suivante, suscitant l'intérêt des maisons d'édition ainsi que de la presse ${ }^{1}$ et nommée par la critique : littérature « de.s banlieue.s », « des cités » et « urbaine » (Chaulet Achour 2005, Cello 2015, Horvath 2007, Vitali 2011, Reeck 2011).

3 Cette relation de complémentarité entre un fait historique, social et la production romanesque, a aussitôt été saisie par les maisons d'édition qui se sont intéressées davantage à ces productions littéraires comme en témoignent les parutions immédiates dans des catalogues renommés comme ceux de Gallimard (Dit violent), Hachette (Du rêve pour les oufs et Dembo story), Lattès (Le poids d'une âme) ou Stock (Cités à comparaître, Supplément au roman national et Chroniques d'une société annoncée). À ce propos, Jean-Marc Roberts, écrivain et directeur des Éditions Stock à l'époque, affirmait que : «Depuis les émeutes, près d'un texte sur cinq que je reçois est l'œuvre d'un jeune de banlieue » (Roberts 2006). 
4 Il faut aussi souligner que l'inévitable concentration sur l'actualité des questions sociales, a eu comme effet un accroissement des critiques ayant une portée plus sociologique. Dans un article de 2008 Olsson confirme cette thèse :

Les soulèvements populaires dans les banlieues de nombreuses villes françaises en novembre 2005 ont sans doute attiré l'attention sur la condition sociale de la population issue de l'immigration. L'orientation des articles sur les auteurs beurs publiés en 2006, où l'actualité des questions sociales apparait comme la raison même des journalistes d'écrire, confirme cette hypothèse. (Olsson 2008)

5 L'ancrage si marqué dans ces territoires, renvoie à une paratopie spatiale mais aussi identitaire «des banlieues ». C'est la condition de l'énonciation et ce n'est qu'à travers elle, que l'œuvre peut advenir. Mais, paradoxalement, elle est rejetée par les auteurs puisqu'elle produit comme effet un éloignement vers les marges du champ littéraire contemporain.

6 À travers l'analyse de six romans, tous parus en 2006: Cités à comparaître de Karim Amellal, Du rêve pour les oufs de Faïza Guène, Banlieue Voltaire de Didier Mandin, Le Poids d'une âme de Mabrouk Rachedi, Dit violent de Mohamed Razane et Banlieue Noire de Thomté Ryam, nous souhaitons proposer une analyse de la représentation littéraire contemporaine de ces lieux aux marges et marginalisés. Nous rassemblons ces romans sous une même étiquette afin de nous interroger sur sa pertinence. En effet, les actions, le décor mais surtout les sujets des narrations s'ancrent dans les quartiers les plus sensibles du tissu urbain, mettant en relief la situation dans laquelle vivent certains jeunes, leurs conditions de vie et leurs relations interpersonnelles.

7 De plus, les auteurs sont nés, vivent ou ont vécu dans ces quartiers, et cela leur donne une connaissance plus tangible des sujets qu'ils abordent. Ce n'est pas un hasard si, exception faite de Didier Mandin, les auteurs ont été les fondateurs en 2007 du collectif littéraire Qui fait la France ?, dont les membres se considèrent comme les « fils de ces émeutes ${ }^{2}$ » et ont publié un recueil de nouvelles intitulé Chroniques d'une société annoncée (2007).

En se proposant comme des romanciers appartenant à « la marge », les auteurs remettent en question le jugement qui a été porté sur eux comme étant ontologiquement à la marge : une littérature marginale, reléguée à la périphérie de la littérature, bref une subculture. Les romans en question se révèlent être d'une marge qui est à entendre comme un espace culturel d'opposition où évolue celui qui témoigne " contre » et d'où émerge le cri rebelle et/ou résilient de ceux qui formulent des expressions de la contestation et de la dissidence.

9 Une particularité de ces textes dérive donc de ce que Melliani (2000) appelle "métissage linguistique ", pour laquelle, dans les banlieues, nous serions face à une combinaison de différents phénomènes langagiers parmi lesquels : l'alternance de langues, la création de formes hybrides, le verlan, ou encore de procédés formels de formation lexicale. Cette forme de langage ne concerne pas tous les écrivains dont nous nous occupons et il n'y a pas une grande nouveauté dans l'usage de la langue du peuple dans la narration. Pourtant, dans le cadre de notre corpus, le sujet n'est pas seulement le peuple décrit par quelqu'un appartenant à un autre "champ social», celui des "dominants", pour reprendre la notion de Bourdieu, comme c'était le cas de Céline dans Voyage au bout de la nuit (Céline 1932), appartenant à une famille de la petite bourgeoisie de la fin du XIX siècle. À l'inverse, c'est le peuple en tant que « dominé » qui parle. L'écrivain, depuis sa posture mal acceptée, mais pourtant authentique d'écrivain « des banlieues », connaît et transfère les codes de cette langue : « Pour nous c'est extrêmement important de se servir 
des mots comme des instruments d'une volonté de contestation ", explique l'écrivain Karim Amellal (2010).

10 Ce qui distingue ces narrations est la plongée dans la vie de la cité3, celle où, malgré les représentations médiatiques, vivent tranquillement des gens ordinaires. Dans notre étude nous questionnerons les représentations de l'espace des protagonistes, en nous demandant : quelles perceptions des lieux en marge ressortent des romans ? Y aurait-il des similitudes avec la vision d'un no man's land et l'émergence d'une "ghettoïsation " crainte par certains sociologues et urbanistes ${ }^{4}$ ou, au contraire, ces romans présentent-ils des dissemblances par rapport à cet imaginaire catastrophique ?

11 Tout d'abord nous analyserons l'approche critique liée à la perception d'une ville dystopique, vision qui conditionnerait la vie des protagonistes. Ce sera l'occasion de nous interroger sur la façon dont l'espace de la cité, également perçu comme un lieu sûr, identitaire, conditionne la trajectoire des personnages dans ces fictions. Ensuite, nous nous proposons de renverser ce schéma dans le but de dévoiler d'autres images qui s'éloignent de celles apocalyptiques présentées auparavant et qui, au contraire, contribuent à recadrer la perception souvent stéréotypée des banlieues.

\section{Vers une image dystopique de la ville}

12 Décider d'ancrer un roman dans le contexte des banlieues sensibles implique certainement de donner à voir une représentation de cette forme d'urbanisme, mais cela relève aussi d'une volonté plus ample, celle de décrire l'histoire d'un personnage en rapport avec son territoire et sa société à un moment précis. Les romans en question sont, sans aucun doute, reliés entre eux par l'environnement géographique des quartiers qu'ils évoquent: les espaces urbains et les immeubles HLM où se déroule la plus grande partie de l'action. Ce qui ressort davantage de cet ancrage spatial est la perception de la banlieue comme espace clos, nous renvoyant à d'autres exemples, bien connus dans le panorama artistique, dont la célèbre citation d'un des protagonistes du film La Haine (Kassovitz 1995) : "On est enfermés dehors. » Ce sentiment psycho-physique apparaît, en effet, comme un mantra, dans la plupart de la production narrative « des banlieues » (Cello 2015, 2016).

13 Cela dit, nous constatons également à plusieurs reprises la présence d'un rapport ambivalent avec cet espace, connoté par une relation dichotomique portant sur l'appartenance - «nous / vous»-, sur l'espace - «dedans / dehors»- ainsi que sur la lumière - « obscurité / lumière ». Tout d'abord, parmi les lieux sombres, internes, clos qui sont narrés, nous trouvons les caves et les prisons. Les premières reviennent constamment dans les romans. Il suffirait de citer le texte précurseur de Rachid Djaïdani, Boumkoeur (1999), dont le titre renvoie à la cave où se déroule l'action. Ces endroits en dessous des tours constituent les décors les plus funestes et tragiques des narrations, puisqu'ils sont à l'abri de la lumière et surtout du regard provenant de « l'extérieur ». Ce sont donc des lieux cachés où adviennent les pires événements, comme en témoigne le récit enchâssé d'Aïcha, la sœur de Zacarias dans Dit violent, lorsqu'elle avoue le viol subi dans une tournante par sept garçons qui l'ont prise par surprise dans le sous-sol de son immeuble. 
de la conséquente division entre les groupes humains est très net: Silou, le protagoniste de Cités à comparaître, le confirme, comparant métaphoriquement à son tour la vie des habitants des cités à celle des animaux : « La faune de la cité me fait penser à Jurassic Park ou bien à des rats vivant dans les égouts du sous-sol »; « On vit comme des rats et les rats ça dit rien à personne » (Amellal $2006: 14,61)$.

18 De plus, ces instances «séparatistes" semblent parfois se prolonger au travers d'une dichotomie entre Blancs et Noirs liée aux origines, par exemple au moment où le narrateur de Banlieue noire accuse « les blancs » d'être en position dominante : «ce sont eux, en majorité, qui réussissent et nous, les plus foncés, qui échouons » (Ryam 2006 : 29). Le protagoniste réitère ici les accusations d'un processus colonial qui, à son avis, n'aurait jamais cessé d'exister: "Ce sont eux qui pillent nos richesses en Afrique et nous qui subissons. Ce sont eux qui vivent dans les beaux quartiers et nous dans les endroits sordides » (Ibid.).

19 Ces fictions nous révèlent ainsi une société imaginaire dystopique, organisée en deux pôles opposés où l'individu se situerait en position de dominé, écrasé par la structure même d'une société dominante l'empêchant de monter l'échelle sociale et d'atteindre le bonheur. Dans la réalité, il est intéressant de se référer à ce que l'urbaniste italien Bernardo Secchi (2013) a récemment exprimé à propos du territoire hexagonal en travaillant au projet $\mathrm{du}$ Grand Paris. Dans son essai, Secchi souligne les fortes responsabilités des politiques de l'urbanisme dans l'aggravation des inégalités sociales, jusqu'à invoquer, lui aussi, la présence d'une nouvelle « question urbaine » (Secchi 2013 : 5). Il démontre la présence d'une sévère injustice spatiale en remarquant que, dans certains territoires français, dont les banlieues, des éléments de l'espace empêchent l'établissement de relations humaines simples et fluides. Ce sont ce qu'il appelle les « zones de Lucifer », où les habitants n'auraient pas les mêmes droits de citoyenneté que ceux vivant ailleurs. Par la suite, il avance une comparaison entre les beaux quartiers du 
sud-ouest parisien et ceux de la Seine-Saint-Denis au nord-est de Paris en discernant une topographie sociale toujours plus contrastée et polarisée.

Il est intéressant de noter la manière dont ce constat urbanistique rejoint les critiques qui se situent à l'intérieur des romans. Prenons encore l'exemple dans Dit violent, où le protagoniste reproche la différence de qualité de vie entre la banlieue où il vit et le centre-ville : « On cherche à reléguer la misère loin de Paris, dans la lointaine banlieue en vérité. On ne peut plus la voir » (Razane 2006 : 77). De façon ironique, le protagoniste de Banlieue noire dénonce cette disparité dans une France divisée en deux, faisant allusion à la chanson La France interprétée par Michel Sardou idolâtrant toute la nation «sauf la nôtre » affirme-t-il (Ryam 2006 : 66). Le protagoniste d'un autre roman, Banlieue Voltaire, évoque la problématique de la distance prenant en compte les infrastructures et les services :

[...] Morcy c'est la problématique de la distance : on est proche de la Capitale, mais on reste trop loin de tout et pas près d'y arriver. Cette distance, je la cite, «c'est celle qui est tout juste suffisante pour empêcher d'aller au théatre ou au resto le soir à Paris, de peur de rater ou de prendre le dernier RER ». (Mandin 2006 : 9)

Toutefois, cette critique n'est pas nouvelle dans le panorama littéraire français. La réalité de ces sites a motivé une réflexion critique chez d'autres auteurs tels que Jean Rolin qui, dans son livre Zone (1995), se promène dans les marges de Paris en passant par BoulogneBillancourt, Clichy-Sous-Bois, Sarcelles, Garges-les-Gonnesse, Nanterre, etc., et en rendant compte de ce qu'il voit et des personnes qu'il rencontre. Nous pouvons prendre aussi l'exemple de J. M. G. Le Clézio qui, déjà dans les années 1980, dans Ariane (1982 : 82), raconte la solitude régnant dans la cité HLM ressemblant à une "ville désertée ", anonyme. Cette façon de présenter la banlieue ne se distingue pas non plus de celle de François Bon dans Décor ciment (1988), roman se déroulant à Bobigny, dans la banlieue de Paris. L'auteur se concentre sur l'état d'abandon et de dégradation des édifices, des parkings et des aménagements routiers inutiles et inutilisés, formulant une sorte de dénonciation de leur existence et une « théorisation » des problèmes qui leur sont liés.

La même critique est reprise par nos romanciers: ils s'en prennent à une situation d'immobilité, de paralysie, qui peut métaphoriquement renvoyer au monde biblique, encore une fois décrit de façon dualiste par l'opposition entre paradis et enfer. C'est ainsi que Silou raconte sa cité, qu'il rapproche du règne du diable puisque le paradis est trop loin : «Mais le paradis y a rien à faire il est trop loin de la cité. Nous y a que le diable qui nous sourit. On est dans son royaume» (Amellal $2006: 83$ ).

Tous les protagonistes de ces romans soutiennent que, pour eux, il n'y aura jamais une possibilité de fuite ou bien de changement, d'amélioration dans l'avenir : « Et comme on a pas d'autre choix que d'être des gladiateurs, notre sang on le foutra un jour dans le biberon des gosses qui grandiront ici» (Amellal 2006: 138). Pourtant, si chez Bon, Le Clézio ou Rolin c'est plutôt le regret d'un passé désormais disparu qui est mis en lumière - celui du peuple des artisans, des ouvriers, des prolétaires qui autrefois « réchauffaient les faubourgs par leur solidarité » (Rubino 2002: 62) -, nos écrivains dévoilent un autre aspect qui se révèle être antithétique par rapport à l'exclusion ressentie: il s'agit de l'affection qu'ils éprouvent pour le quartier. En effet, il apparaît que les liens se défont à mesure que se tissent d'autres sentiments établis sur une forte identification et appartenance à la cité. 


\section{« Ici, on est chez nous » : I'identification au quartier} immeubles et moi » (Ryam $2006: 147$ ). Quand il rentre dans son quartier, il se sent à l'abri,
chez lui : « comme une pierre en Palestine, ou une bouteille de vodka à Moscou » (Ryam $2006: 35)$.

Le sentiment si marqué envers sa propre cité constituerait, en quelque sorte, le contrepoids de l'exclusion ressentie. Le quartier, et plus précisément les lieux publics - la place principale en bas des tours, qui en constitue le cœur - sont perçus par Mehdi comme des lieux sûrs, protégés, auxquels il appartient :

C'est là en bas de la tour, que se racontent les dernières embrouilles [...] C'est un lieu rassurant, il y a toujours quelqu'un. C'est là que s'est constitué le clan, une horde formée de tous les jeunes du quartier qui traînent avec une amertume, une haine, mais aussi une folle envie d'exister. (Razane 2006 : 79)

Ce sentiment aux contours flous donne naissance à la perception d'un territoire précis et délimité, qui présuppose des frontières : celles du quartier. Pour les jeunes protagonistes de ces romans, ce dernier ressemble à un lieu vaste mais en même temps intime, où les relations sont vives et où " chacun se connaît » et se sent chez lui. Bref, il s'agit d'un lieu familier à l'intérieur duquel se développe une forte sociabilité et où les relations entre amis acquièrent une importance radicale.

De ce qui précède, nous notons la façon dont la conception de l'espace est perçue à double vitesse, chargée de plusieurs valeurs. En effet, bien qu'il soit considéré comme lieu de confinement social et territorial, discrédité, décrit comme malpropre, sale, dangereux, où l'ennui règne, l'espace de la cité est finalement perçu comme rassurant grâce aux solidarités internes et, de ce fait, il devient pénible de le quitter. Les raisons de cet attachement naissent justement de ce sentiment d'amour-haine, puisqu'il s'agit d'un lieu rassurant, connu, à l'inverse de ce qui se trouve au-delà du périphérique. Cette adhésion au lieu familier est amplifiée en cas de risque ou bien de menace provenant «de l'extérieur ", soit de la part des forces de l'ordre, soit de celle des bandes des quartiers voisins, qui sont considérées comme persona non grata dans les quartiers, comme des intrus dans ce que les jeunes considèrent être « leur territoire».

La représentation des banlieues est-elle univoque, ou y a-t-il des aspects antinomiques repérables dans les récits objet de notre étude ? Au-delà des images de l'espace que nous venons de souligner, nous rechercherons par la suite la présence de perceptions spatiales alternatives.

Pour ce faire, nous nous appuyons sur un article de Bruno Levasseur (2008) dans lequel, tout en entamant une comparaison entre les productions cinématographiques et documentaires des années 1990 ayant comme cadre la banlieue, il explique comment ces dernières proposent des représentations plus proches de la banalité et de la vie quotidienne, contrairement à l'imaginaire commun et médiatique renvoyant souvent à une nation française désintégrée, dont le problème et le péril des banlieues seraient le cœur. En partant de cela, nous vérifierons la présence dans les romans en question d'autres exemples qui s'inscrivent le plus possible dans la représentation de l'authenticité du contexte des cités françaises. 


\section{Pour une réhabilitation de l'image des banlieues}

30 Au début des années 1990 se fait jour une nouvelle image de la banlieue à travers les documentaires, sensibilisant les spectateurs au côté quotidien, ordinaire des relations humaines dans les quartiers défavorisés. Le but des réalisateurs est de dépasser les images stéréotypées caractérisées par le sentiment général d'alarme invoqué par le cinéma et les médias (Berthaut 2013). Il n'est pas anodin que l'apparence de cette communauté des cités apocalyptiques à la dérive, livrées à l'abandon, à la violence et à la guerre entre bandes et forces de l'ordre ait été révélée dans les mêmes années par le «cinéma de banlieue » et son "retour au réalisme » (Powrie 1999) à travers un film emblématique comme La haine. Ces films ont été critiqués pour leur tendance sensationnaliste, où la violence apparaît comme exotique et le conflit et la peur sont poussés à l'extrême. Au contraire, dans les documentaires, l'accent se déplace vers ce qu'il y a de plus insignifiant et « banal » (Billing 1995) dans ces endroits, comme la queue au bureau de poste (Une poste à La Courneuve de Dominique Cabrera, 1994) ou bien les difficultés liées à la rénovation urbaine ( $L a$ ville est à nous de Patrick Laroche, 2000), pour inclure les interactions quotidiennes des habitants faisant par exemple les courses au supermarché.

Plusieurs traits d'union lient ces documentaires aux romans pris en compte dans cette étude, dans lesquels nous pouvons repérer d'autres perceptions de l'espace, qui se situent bien loin du sensationnalisme et de la violence. En effet, contrairement à ce que l'on pourrait penser, les exemples d'apparente banalité et de quotidienneté y sont nombreux. Les scènes de la vie habituelle ainsi que le développement des sentiments amoureux, les cas de réussite scolaire ou bien sportive, appartiennent à une normalité presque insignifiante et ordinaire qu'il serait injuste d'oublier au profit des perceptions alarmistes. Pensons tout d'abord à Le poids d'une âme où le personnage Tarik, se déplace d'un lieu à un autre pour accomplir les tâches routinières: les courses, la poste et la pharmacie.

Sécurité sociale, Trésor public, mairie, pharmacie, bureau de Poste, supermarché, Tarik s'est multiplié pour satisfaire les exigences familiales. À part un contretemps aux impôts et une queue monstre à La Poste, la routine (Rachedi $2006: 40$ ).

Le quotidien de ce jeune homme est caractérisé par des actions habituelles auxquelles il se dédie au profit de toute sa famille qui bénéficie ainsi de sa connaissance de la langue et de la bureaucratie françaises, ses parents étant des Maghrébins presque analphabètes. Sa vie est celle d'un individu anonyme qui passe inaperçu, n'ayant rien de captivant par rapport aux conduites violentes, aventureuses et sensationnalistes des autres personnages. Toutefois, il incarne un aspect jamais mis en avant, celui d'une relation à l'espace normale, quotidienne, ne possédant rien de surprenant, exotique ou spectaculaire qui s'établit dans une banlieue quelconque, qui nous pousse à réfléchir sur la diversité qui pourtant demeure dans ces lieux.

Les textes sont aussi riches en scènes ordinaires, comme le rendez-vous assez banal d'un couple au café situé au-delà du quartier : «sur le chemin on s'est rien dit. [...] Elle était trop belle. [...] J'ai enlevé ma casquette quand on est sorti de la cité » (Amellal $2006: 145$ ). Ce geste est significatif puisqu'il démontre qu'en enlevant sa casquette, c'est comme si Silou se dépouillait de son appartenance banlieusarde, accédant ainsi à un contexte émotionnel différent qui n'est pas celui de sa vie quotidienne brutale. Le sentiment amoureux fonctionne ici comme contrepoids à la vie rude et violente. Prenons aussi 
l'exemple de Mehdi, le protagoniste de Dit violent, qui s'éprend de Marie, une jeune enseignante en sociologie ; au fil du roman, un lien se constitue entre l'amour pour elle et le monde artistique auquel elle l'initie. Ces deux nouveaux univers opèrent une transformation dans l'âme de Mehdi. En même temps que l'amour, il découvre le théâtre. Grâce à la femme aimée, la "Shéhérazade » de son " pays de Mille et une Nuits, des Mille et Un Tourments » (Razane $2006: 63$ ), il découvre des artistes impliqués et, en entrant en relation avec eux et leur création artistique, Mehdi s'empare de valeurs qui ne lui appartenaient pas. Il nous semble que l'auteur veut ainsi briser le stéréotype de la représentation typique du jeune de banlieue. Afin de dépasser cette représentation sombre de la banlieue, l'auteur crée aussi le personnage du poète Zacarias, ami de Medhi, dont les mots sont dotés du pouvoir non seulement d'embellir le monde, mais aussi de séduction. Le rapport antithétique entre violence et poésie est particulièrement frappant dans les parties du roman où apparaitt la langue de Zacarias qui est teintée de poésie.

Nous remarquons aussi dans d'autres romans que, contre l'image du jeune paresseux, violent et délinquant, on présente des exemples de réussite scolaire ou bien sportive. Tel est le cas de Ludo, le héros de Banlieue Voltaire, qui obtient son bac scientifique avec mention très bien.

Dans Banlieue noire, les deux sœurs du protagoniste réussissent dans leurs études, malgré l'abandon du père et la pauvreté de la mère, s'installant à Paris pour fréquenter l'université. Voici comment leur frère, qui a choisi de rester dans le quartier, les décrit: «Maé, dix-neuf ans prépare un Deug de Droit, et Hébi, vingt ans, une licence de Maths. Elles passent leur temps à taffer, comme si elles aimaient ça » (Ryam 2006 : 91).

Une dernière remarque concerne la présence que ces romans accordent à l'État. En effet, contrairement aux représentations d'un No man's land que les institutions nationales auraient définitivement abandonné en raison d'un danger croissant, ces dernières sont bien présentes dans les romans, non seulement à travers les établissements scolaires, mais aussi les forces de l'ordre et surtout les services sociaux. Les exemples de la présence de ces derniers sont nombreux et elle se concrétise par l'activité des assistants sociaux dans les écoles, par la description de la queue devant les guichets de la Caisse d'allocations familiales ou encore par les fréquentes évocations de l'ASSEDIC et de l'ANPE ${ }^{5}$ , de la Poste ou des transports publics tels que la RATP, qui marquent la présence des services fondamentaux de l'administration attestant l'intégration de ces habitants dans la société.

\section{Conclusion}

Nous avons constaté que l'espace de la cité conditionne la trajectoire des personnages dans ces fictions, parfois conçues mais aussi perçues comme des "documents ", grâce à leur «fidélité au réel » pour mieux donner à comprendre la vie des habitants des cités. Cela s'insère dans la volonté de susciter le réveil de la conscience publique de la nation, souhaité par les auteurs de ces romans.

Bien que dix ans soient passés depuis la publication de ces récits, les propos et les préoccupations abordées restent très actuels. Le souci de la "question urbaine ", des marges où subsistent des difficultés d'intégration est malheureusement encore tangible. Pourtant, il serait inexact de parler d'une supposée "ghettoïsation » des banlieues que redoutent parfois les sociologues et les urbanistes. La situation est tellement hétérogène 
et délicate qu'il est nécessaire de discerner finement les dynamiques présentes dans ces lieux. Les exemples « insignifiants » de la représentation d'une apparente "normalité » sociale, que nous venons d'analyser dans les narrations, contribuent à attirer l'attention sur ce qui passe souvent inaperçu dans ces territoires, mais qui pourtant existe : l'activité ordinaire de ses habitants.

En effet, la narration de ces détails, de ces petits errements spatiaux des personnages, nous introduit à ce que le critique Dominique Viart (2011) nomme une nouvelle forme d'« humanisme » dans la littérature de l'extrême contemporain, pour se référer non plus à la foi aveugle dans la grandeur des aventures de l'homme, mais à ses faiblesses et à ses errements. C'est à ce propos qu'il parle d'« éthique de la minutie » (Viart 2004:61), c'està-dire du goût narratif d'aller vers le plus petit. C'est l'intérêt littéraire pour le détail, comme nous l'avons vu dans les exemples cités, minimes, mais très significatifs. Bien qu'elle soit fictive, cette apparente «insignifiance », participe à recadrer la perception des banlieues.

Ainsi, de la même façon que les documentaires cités ont participé à la réintégration des cités et de leurs habitants dans l'imaginaire des spectateurs des années 1990, d'un point de vue social, politique, culturel et spatial, ces représentations narratives présentent, à leur tour, des alternatives. En dévoilant ce qu'il y a de plus factuel, complexe et subtil dans la quotidienneté de ces territoires, elles ont le mérite de réorienter l'image couramment proposée d'une banlieue dangereuse et violente, stimulant un débat et une réflexion la plus ample et approfondie possible, en permettant de repenser la situation et la question des habitants des périphéries françaises, en les montrant non pas comme à l'écart, « à part », mais en tant que « part » de la communauté nationale.

\section{BIBLIOGRAPHIE}

Amellal, Karim et Razane, Mohamed, 2012, "Qui fait la France », Radar-Musac's journal of art and thought, vol. 1, p. 72-88.

Berthaut, Jérôme, 2013, La Banlieue du 20 heures. Ethnographie de la production d'un lieu commun journalistique, Marseille, Agone.

Berthod, Anne, 2006, « La banlieue a du style », L'Express, [En ligne], http://www.lexpress.fr/ culture/livre/du-reve-pour-les-oufs-pieds-blancs-le-poids-d-une-ame-desintegration-cites-acomparaitre_821691.html.

Billing, Michel, 1995, Banal nationalism, Londres, Sage.

Bourdieu, Pierre, 1993, La Misère du monde, Paris, Seuil.

Chaulet Achour, Christiane, 2005, « Banlieue et littérature », dans M.-M. Bertucci et V. HoudartMerot (dir.), Situations de banlieue - Enseignement, langues, cultures, Lyon, INRP, coll. « Éducation, politiques, sociétés ", p. 129-150.

Cello, Serena, 2015, « Pour une narration des banlieues contemporaines », Roman 20-50, n 59, p. 167-176. 
Cello, Serena, 2016, « La jeunesse de rue : quelles représentations littéraires contemporaines?», dans M. Boucher (dir.), Jeunesses de rue. Représentations, pratiques et réactions sociales, Paris, L'Harmattan, p. 53-68.

Donzelot, Jacques, 2006, Quand la ville se défait. Quelle politique face à la crise des banlieues ?, Paris, Seuil.

Horvath, Christina, 2007, Le Roman urbain contemporain, Paris, Presses Sorbonne Nouvelle. Levasseur, Bruno, 2008, « De-essentializing the Banlieues, Reframing the Nation: Documentary Cinema in France in the late 1990s ", New Cinemas: Journal of Contemporary Film, vol. 6, nº 2, p. 97-110.

Maurin, Éric, 2004, Le Ghetto français. Enquête sur le séparatisme social, Paris, Seuil.

Melliani, Fabienne, 2000, La langue du quartier, Paris, L'Harmattan.

Olsson, Kenneth, 2008, «L'effet beur. La littérature beure face à la réception journalistique », Expressions Maghrébines, revue de la CICLIM, vol. 7, nº 1, p. 104.

Powrie, Phil, 1999, « Heritage, History, and New Realism », dans P. Powrie (dir.), French Cinema in the 1990s: Continuity and Difference, New York, Oxford University Press, p. 1-21.

Reeck, Laura, 2011, Writerly identities in Beur Fiction and Beyond, Lanham, Lexington Books.

Rubino, Gianfranco, 2002, «L'envers du lieu : Bon, Daeninckx, Rolin, Toussaint », Lendemains, vol. 27, nº 107-108, p. 56-72.

Secchi, Bernardo, 2013, La città dei ricchi e la città dei poveri, Bari, Laterza.

Viart, Dominique, 2004, « Le moindre mot : Pascal Quignard et l'éthique de la minutie », Revue des sciences humaines, $\mathrm{n}^{\circ} 260$, p. 61-73.

Viart, Dominique, 2011, « Littératures au pluriel : entretien avec Dominique Viart » par Bruno Blanckmann, Raison-publique.fr, 24 janvier 2011, [En ligne], http://www.raison-publique.fr/ article400.html, consulté le 16 novembre 2015.

Vieillard-Baron, Hervé, 2006, « Des banlieues françaises aux périphéries américaines : du mythe à l'impossible confrontation?", Hérodote, $\mathrm{n}^{\circ} 122$, p. 10-24.

Vieillard-Baron, Hervé, 2011, Banlieues et périphéries. Des singularités françaises aux réalités mondiales , Paris, Hachette.

Vitali, Ilaria, 2011, Intrangers : post-migration et nouvelles frontières de la littérature beur, t. I, Bruxelles, Éditions Academia.

Vitali, Ilaria, 2010, “ “À l'avant-garde du réel” entretien avec Mohamed Razane et Karim Amellal du collectif Qui fait la France? », Francofonia, n 59, p. 121-130.

Wacquant, Loïc, 2006, Parias Urbains. Ghetto, banlieue, État, Paris, La Découverte, p. 145-171.

\section{Corpus}

Amellal, Karim, 2006, Cités à comparaître, Paris, Stock.

Guène, Faïza, 2006, Du rêve pour les oufs, Paris, Hachette Littératures.

Mandin, Didier, 2006, Banlieue Voltaire, Fort-de-France, Desnel.

Rachedi, Mabrouck, 2006, Le Poids d'une âme, Paris, Lattès. 
Razane, Mohamed, 2006, Dit violent, Paris, Gallimard.

Ryam, Thomté, 2006, Banlieue noire, Paris, Présence africaine.

\section{Romans}

Céline, Louis-Ferdinand,Voyage au bout de la nuit, Paris, Delanoël, 1932.

Bon, François, 1988, Décor ciment, Paris, Minuit.

Djaïdani, Rachid, 1999, Boumkoeur, Paris, Seuil.

\section{NOTES}

1. Voir par exemple : Anonyme, «La littérature française prend ses couleurs ", Elle, 4 septembre 2006, p. 261 ; Selim Ardaoui, "Y-a-t-il une littérature de banlieue», Presse\&cité, 12 septembre 2009, [En ligne], http://www.presseetcite.info/journal-officiel-des-banlieues/culture/y-t-il-unelitterature-de-banlieue, (consulté le 12 juillet 2016) ; Sarah Adams, "Voice of the people ", The Guardian, 10 mai 2006, [En ligne], http://www.theguardian.com/society/2006/may/10/ books.socialexclusion (consulté le 20 mars 2016); François Cyrille, «Des littératures de l'immigration à l'écriture de la banlieue ", Synergies Sud-Est européen, $\mathrm{n}^{\circ} 1$, 2008, p. 149-157 ; Réjane Éreau, «La littérature prend l'air et le RER », Respect Magazine, $\mathrm{n}^{\circ}$ 13, janvier-mars 2007, p. 30-32 ; Faïza Guène, «Interview de Faïza Guène, Prendre la parole », par Mélanie Carpentier, Evene.fr, 17 août 2006, [En ligne], http://www.evene.fr/livres/actualite/interview-faiza-guenereve-pour-les-oufs-kiffe-demain-440.php (consulté le 12 juillet 2013) ; Sabrina Kassa, "Écrivain de banlieue, ça n'a pas de sens!", Africultures, 25 juin 2008, [En ligne], http:// www.africultures.com/php/?nav=article\&no=7690, consulté le 10 octobre 2014 ; Céline Ngi, « La nouvelle littérature urbaine", [Indisponible], http://www.fluctuat.net/6451-La-nouvellelitterature-urbaine, consulté le 25 septembre 2014; Steve Puig, «Interview avec Mohamed Razane », Expressions Maghrébines, vol 7, n 1, 2008, p. 86 ; Elsa Vigoureux, «Banlieue : la nouvelle vague littéraire ", Le nouvel observateur, 27 juillet-2 août 2006, p. 73.

2. "Qui fait la France ", Radar-Musac's journal of art and thought, vol. 1, 2012, p. 72-88. "We are in a sense the children of those riots - or of that revolt. Moved and made indignant by the fate of these lost territories, which have been neglected by the state and public authorities for decades, we could not sit idly by and watch from our ivory towers on the pretext that we-writers, artists, teachers-had ourselves managed to escape the ghetto by the work of our pens and minds [...] we were of course aware of the underlying causes of these protests and this violence. Having experienced them ourselves, we understood their workings and motivations, even if the form of expression did not strike us as the most pertinent or likely to achieve anything " (Amellal et Razane 2012, traduction de Serena Cello). «Nous sommes en quelque sorte les fils de ces émeutes - ou de cette révolte. Touchés et indignés par le destin de ces territoires perdus, qui ont été négligés par l'État et par les autorités publiques pendant des décennies, nous ne pouvions pas rester assis passifs et regarder de notre tour d'ivoire, sous le prétexte que nous - écrivains, artistes, enseignants - nous avions essayé de fuir du ghetto grâce au travail de nos plumes et de nos esprits [...] nous étions bien sûr conscients des causes sous-jacentes de cette protestation et de cette violence. L'ayant expérimentée nous-mêmes, nous avons compris leurs gestes et leurs motivations, bien que la forme d'expression utilisée ne soit pas la plus pertinente ou plausible pour atteindre quoi que ce soit. "

3. Nous nous référons à la cité dans le contexte des quartiers sensibles, comme un ensemble d'immeubles formant un tout homogène d'un point de vue architectural, social, à l'intérieur du 
quartier urbain défavorisé. Voir la définition du dictionnaire Larousse 2013 : « Nom donné à des ensembles d'immeubles, de maisons formant un tout homogène ou ayant une même destination. »

4. Voir à ce propos Loïc Wacquant (2006). Voir les constats convergents d'Éric Maurin (2004), Hervé Vieillard-Baron (2006) et Jacques Donzelot (2006).

5. Actuel Pôle emploi.

\section{RÉSUMÉS}

À l'intérieur du rapport conflictuel entre centre et marges nous trouvons la «littérature des banlieues » née à la suite des violentes émeutes urbaines de 2005. À travers six romans publiés en 2006 nous nous interrogeons sur la mise en récit des marges urbaines dans un rapport ambivalent entre volonté de sensationnalisme et ordinaire banalité ; des récits qui visent à replacer les marges au centre non seulement de l'actualité, mais aussi de l'univers littéraire, contribuant à apporter de nouvelles perspectives d'analyse aux représentations déjà connues des quartiers sensibles.

Within the conflicted relationship between centre and suburbs known as "contemporary social problem," we find the "banlieue literature" born after French urban disorders in 2005. Throughout six novels published one year later, we question the contemporary literary representation of these places in a problematic correlation between sensationalism and banality. Those texts want to replace margins at the centre of the actual debate as well as the French literature, contributing also to engender other perspective's analysis to the most prevalent ones.

\section{INDEX}

Mots-clés : littérature des banlieues, littérature urbaine, périphéries, quartier sensible, espace urbain

Keywords : French literature, urban literature, suburbs, suburban studies, french banlieues

\section{AUTEUR}

\section{SERENA CELLO}

Université de Gênes et Université François-Rabelais de Tours 